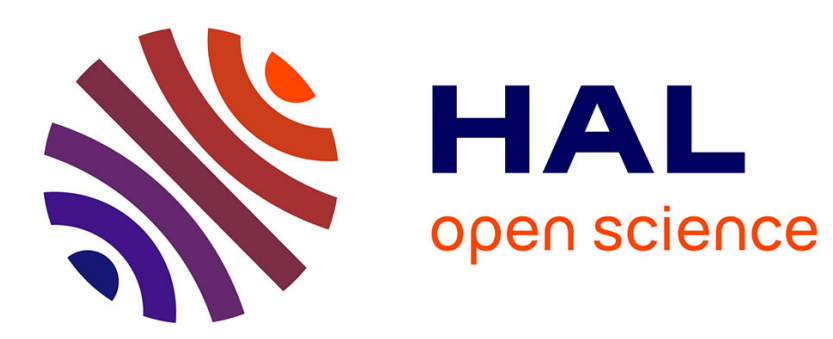

\title{
Télétechnologies et nouvelles configurations psychiques Philippe Givre
}

\section{To cite this version:}

Philippe Givre. Télétechnologies et nouvelles configurations psychiques. Champ Psychosomatique, 2006, www.corps.com, 3 (43), pp.109 - 124. 10.3917/cpsy.043.0109 . hal-01504278

\section{HAL Id: hal-01504278 \\ https://hal.science/hal-01504278}

Submitted on 9 Apr 2017

HAL is a multi-disciplinary open access archive for the deposit and dissemination of scientific research documents, whether they are published or not. The documents may come from teaching and research institutions in France or abroad, or from public or private research centers.
L'archive ouverte pluridisciplinaire HAL, est destinée au dépôt et à la diffusion de documents scientifiques de niveau recherche, publiés ou non, émanant des établissements d'enseignement et de recherche français ou étrangers, des laboratoires publics ou privés. 


\title{
TÉLÉTECHNOLOGIES ET NOUVELLES CONFIGURATIONS PSYCHIQUES
}

\author{
Philippe Givre
}

L’Esprit du temps | «Champ psychosomatique »

2006/3 n 43 | pages 109 à 124

ISSN 1266-5371

ISBN 9782847950922

Article disponible en ligne à l'adresse :

http://www.cairn.info/revue-champ-psychosomatique-2006-3-page-109.htm

\section{Pour citer cet article :}

Philippe Givre, «Télétechnologies et nouvelles configurations psychiques », Champ psychosomatique 2006/3 (n 43), p. 109-124.

DOI 10.3917/cpsy.043.0109

Distribution électronique Cairn.info pour L'Esprit du temps.

(C) L'Esprit du temps. Tous droits réservés pour tous pays.

La reproduction ou représentation de cet article, notamment par photocopie, n'est autorisée que dans les limites des conditions générales d'utilisation du site ou, le cas échéant, des conditions générales de la licence souscrite par votre établissement. Toute autre reproduction ou représentation, en tout ou partie, sous quelque forme et de quelque manière que ce soit, est interdite sauf accord préalable et écrit de l'éditeur, en dehors des cas prévus par la législation en vigueur en France. Il est précisé que son stockage dans une base de données est également interdit. 


\title{
Télétechnologies et nouvelles configurations psychiques
}

\author{
Philippe Givre
}

\section{SUBJECTIVATION ET SUPPLÉMENTS ARTIFICIELS}

$\mathrm{L}$

'enchaînement des mutations techno-scientifiques du siècle dernier nous a propulsé dans un univers de plus en plus sophistiqué où le virtuel prend le pas sur la réalité du monde matériel. Ainsi pour Cassirer, la «dématérialisation» de la technique pourrait bien constituer un des problèmes centraux de notre époque. Dans une perspective assez semblable, Derrida cerne le mal contemporain d'abord en tant que mal d'abstraction, lié à la télé-technoscience ou au «cyberespace». La «crise de la subjectivité » de nos sociétés contemporaines serait ainsi liée aux phénomènes de dissociation et de désincarnation qu'induisent les nouvelles technologies contribuant "à nous séparer de nous même».

Cautionnant également l'antinomie supposée entre subjectivation et monde de la technique, d'autres argumentaires critiques, notamment psychanalytiques et d'obédience lacanienne, dénoncent le plus souvent une mise en péril de la clef de voûte de l'organisation psychique. Ils nous laissent ainsi craindre un vacillement inquiétant de l'ordre symbolique, au profit d'un «symbolique virtuel » promouvant l'effacement de la perte et des interdits fondateurs. De fait, en entretenant la croyance que « tout est possible » ou que « rien n'est impossible », le discours techno-scientifique nous ferait ainsi courir le risque de «décapitonner l'arrimage, pourtant indispensable, de l'interdit dans la catégorie de l'impossible. ${ }^{1} \mathrm{En}$ d'autres termes, le monde techno-scientifique participerait d'une idéologie de l'abolition du sujet.

1. J.P. Lebrun, Un monde sans limite, Erès, 1997, p. 138.

Philippe Givre - Psychanalyste - Maître de conférences - UFR Sciences Humaines Cliniques - Université Denis Diderot - Paris VII. Membre de l'Ecole doctorale de Psychanalyse et de 1'Equipe de recherche sur l'Adolescence. 80 rue de Jemmapes 59800 Lille.

Champ psychosomatique, 2006, $\mathrm{n}^{\circ}$ 43, 109-124. 
2. On ne trouve en effet aucune trace dans les textes de Freud d'une telle défiance face aux progrès de la science et des techniques puisqu'à

l'inverse il a toujours eu le souci de conférer

le statut de science à la psychanalyse, définie elle-même comme nouvelle discipline scientifique.

«La psychanalyse est particulièrement qualifiée pour être

le porte-parole de la conception scientifique de l'univers. »

«D'une conception de l'univers », in Nouvelles

Conférences sur la psychanalyse, Gallimard.

3. Comme l'énonce

J. Kristeva, le narcissisme primaire est édifié autour de deux pôles, celui de

l'identification primaire

à un père imaginaire supposé aimant et, d'autre part, celui de l'imago maternelle archaïque suscitant à la fois fascination et répulsion, désir et haine.

Or, la structuration du narcissisme primaire aurait notamment à pâtir de l'absence d'une variante laïque pour le père aimant, suite à la «mort de Dieu».
S'il ne s'agit pas d'édulcorer les risques qui pèsent sur l'évolution de nos sociétés contemporaines, nous souhaitons nuancer ces discours et appréhender de façon plus modulée les conséquences subjectales du développement en accéléré des télétechnologies ${ }^{2}$. Certes, une approche des modalités de subjectivation ne peut à notre sens ignorer l'existence et l'impact des simulacres technologiques, des images de synthèse, de l'espace virtuel propre aux télétechnologies. Ces nouveaux supports semblent en effet proposer aujourd'hui ce que l'on pourrait assimiler à des suppléments psychiques artificiels qui participent étroitement de la construction et de l'équilibre subjectal des individus. Encore conviendra-t-il de préciser davantage ce que recouvre pour nous cette notion de supplément artificiel qui en faisant office de prothèse psychique favorise l'externalisation ou la «périphérisation » de la vie psychique. Nous aurons dans le même temps à mettre en regard ces phénomènes avec l'impact des processus psychiques et notamment des processus adolescents qui en eux-mêmes génèrent un aménagement singulier des limites entre réalité interne et réalité externe.

Dans la même perspective, nous aurons à montrer le côté simplificateur, sinon réducteur, des approches qui n'entrevoient que le seul impact des nouvelles technologies sur le fonctionnement psychique sans prendre en compte la dynamique inverse, à savoir l'implication et la « responsabilité » des nouveaux modes de subjectivation dans l'avènement de cet univers où les produits et accessoires " high tech» occupent une place de plus en plus prépondérante. En d'autres termes, dans ce monde contemporain où la course à la performance devient une priorité, il s'agit bien d'appréhender quel(s) gain(s) psychique(s) sont susceptibles de générer ces suppléments artificiels. En effet, le souci de performance inhérent à l'univers virtuel pourrait bien refléter une quête, sinon une exigence, de performance propre à l'économie psychique. Loin d'être incidente ou contingente, l'une des visées prioritaires de l'inventivité techno-scientifique pourrait être de répondre à une nécessité interne et subjective, en cherchant d'une part à abaisser le niveau de la dépense psychique pour chaque individu et d'autre part à répondre au déficit de nos sociétés qui ne parviendraient plus à construire de façon satisfaisante le narcissisme primaire ${ }^{3}$.

Si l'objet électif de Narcisse est l'espace psychique lui-même, les difficultés rencontrées dans l'édification du narcissisme vont être directement responsables de la possibilité ou de l'impossibilité pour le sujet de disposer de son espace psychique et de ses capacités de représentation. Ne faudrait-il pas alors concevoir que la propension toujours plus grande à s' harnacher de gadgets «new look » et « high tech» s'évertuerait à contrebalancer le déficit d'intériorité des 
sujets de notre (post)modernité ? De la même manière, le recours systématisé à des flots d'images ne devrait-il pas être envisagé en lien avec le fonctionnement trop discontinu de l'activité de représentation ; discontinuité qui serait secondairement responsable d'un déficit d'intégration des signes iconiques capables d'alimenter l'activité auto-érotique de l'infans. A défaut de disposer de ces signes iconiques capables de nourrir son activité imaginaire et fantasmatique, le sujet cherchera via les images ou de signes sensibles qui émanent des différents supports ou médias télétechnologiques de quoi pallier ces carences qui trouvent leur origine au niveau des interrelations précoces de l'infans avec son environnement primaire. En cela, la pente inflationniste que suit la production de ces nouveaux médiums nous laisse à croire que les besoins ou néo-besoins psychiques, intrinsèquement liés à ces carences narcissiques, pourraient être eux aussi en expansion croissante. Encore faudrait-il préciser la nature de ces néo-besoins et leur rôle au sein de l'économie psychique, sans pour autant sombrer dans la nostalgie «d'un « dedans » plein, réflexif et maître de ses pertes et de ses errances $»^{4}$, puisque nous chercherons davantage à entrevoir ce qui s'augure comme nouveaux modes d'être à partir de ces nouveaux réglages du narcissisme et de ces nouvelles modalités de secondarisation.

\section{VERS DE NOUVELLES MODALITÉS DE SECONDARISA- TION?}

Si Freud évoquait en son temps l'ensemble des prothèses que chaque individu se devait d'abandonner nécessairement au coucher (lunettes, dentier, et autres postiches), l'accroissement exponentiel de ces prothèses artificielles que sont les portables, les I-Pod, clés USB, consoles vidéos, etc. oblige à reconsidérer l'ensemble des processus qui participent de la construction subjective à l'aune de cette révolution culturelle. En effet, les bouleversements induits au niveau des modalités de symbolisation paraissent œuvrer à une transformation significative des caractéristiques inhérentes au processus secondaire. L'une des spécifications majeures de ces modifications viserait à délester le fonctionnement psychique de la pesanteur du registre conceptuel de l'activité psychique pour retrouver, voire inventer des modalités de secondarisation nouvelles et moins contraignantes. On le sait, les processus cognitifs ont des exigences élevées à l'égard du moi quant au degré d'inhibition des motions désirantes sous-jacentes au travail de penser. En cela, le recours aux images virtuelles - que ce soit aux images numériques
4. J. Kristeva, Histoires d'amour, Paris, Denoël, 1983, p. 353. 
5. « Cette formulation, présentation plastique et figurative, traduit le mot allemand Darstellung, terme qui veut dire beaucoup de choses : ça veut dire représentation théâtrale, mais die darstellende Künste signifie aussi les arts plastiques; le terme désigne l'actualisation de quelque chose, par exemple, de la pulsion, et il met en jeu tout ce qui est de l'ordre figuratif et plastique. Autrement dit, une Darstellung, ce n'est pas une Vorstellung. C'est quelque chose qui est de l'ordre de la présentation figurative, ce n'est pas une représentation."

M. David-Ménard, Identification et hystérie, in Les identifications, ouvrage collectif, Paris, Denoël, 1987, p. 84.

6. J.L. Weissberg, Entre présence et absence, un virtuel toujours plus corporel, in le virtuel, la présence de l'absent, ouv. coll. sous la dir. de

$\mathrm{S}$. Missonnier et $\mathrm{H}$. Lisandre, Paris, EDK, 2003, p. 90. ou aux images interactives - déroge à ce niveau d'exigence, puisqu'il permet de retrouver des frayages plus anciens et propres aux images motrices lesquelles traduisent originairement l'expression des motions désirantes du sujet. Ces images actées correspondent d'assez prêt à l'usage que Freud proposait d'un premier niveau de représentation imagée sous le terme de Darstellung qu'il convient de distinguer des Vorstellung. A côté de la Vorstellung, le concept traditionnel dans la philosophie idéaliste, conçu comme copietraduction de perception, la Darstellung 5 (qui se traduit plus justement par mise en présence) recouvre des mouvements, des transformations, déformations des Vorstellung sans qu'on ne puisse déceler la part de l'objet perçu et celle des intensités énergétiques dont le psychisme fait preuve pour les faire exister en eux-mêmes. L'image dans la Darstellung détient sa valeur et sa singularité d'être l'équivalent formel des quantités énergétiques et du polymorphisme sémiotique qui ont présidé à sa présentation. Aussi, en favorisant le commerce avec ces images «à fort coefficient d'incarnation » que sont les Darstellung - c'est-à-dire des images "qui ne sont plus faites pour être vues, mais pour être enchaînées avec des actes ${ }^{6}$ - l'immersion dans la réalité virtuelle empruntent des voies pas tout à fait inconnues pour le fonctionnement psychique.

Par ce biais, il s'agit bien en effet d'imposer un détour et un différé de la satisfaction pulsionnelle moins conséquent que celui réclamé par les processus de penser. En se situant à mi-chemin entre identité de pensée et identité de perception, ces voies nouvelles privilégient le recours à la motricité et aux images pour mieux s'émanciper d'un fonctionnement psychique rationnel et intellectualisé, tout en restant néanmoins dans le registre de la pensée logique et de l'action contrôlée. Mais surtout, elles permettent de nourrir les processus secondaires qui trouvent là une matière figurable capable de renforcer le potentiel métaphorique des énoncés langagiers. En d'autres termes, le pôle figuratif intrinsèque aux images-mouvements des jeux vidéo de part ses alliances avec les motions désirantes sous-jacentes à la présence des Darstellung redonne une consistance affective, imaginaire à un discours menacé de fonctionner sur un mode ascétique et intellectualisé. Bien sûr nous concèderons volontiers que la richesse métaphorique des mythèmes des jeux vidéo s'avère toute relative, voire assez limitée.

En effet dans la perspective deleuzienne, la narration et plus encore sous sa forme moderne est fondée dans l'image même, elle découle des images-mouvements ou de leur spécification en imagesperception, images-affection, images-pulsion, images-action. Or, l'image-mouvement est une matière non linguistiquement formée. «C'est une masse plastique, une matière a-signifiante et a- 
syntaxique, une matière non linguistiquement formée, bien qu'elle ne soit pas amorphe et soit formée sémiotiquement, esthétiquement, pragmatiquement. C'est une condition, antérieure en droit à ce qu'elle conditionne. Ce n'est pas une énonciation, ce ne sont pas des énoncés. C'est un énonçable. ${ }^{7} \mathrm{C}^{\prime}$ 'est de cette matière, de cette masse plastique que le langage s'empare pour former des énoncés qui vont dominer ou remplacer les images. La langue n'existe donc que dans sa réaction à une matière non-langagière qu'elle transforme. Il n'est d'ailleurs pas anodin que Deleuze emprunte à Eisenstein la notion d'idéogramme pour caractériser cette masse plastique, cette matière a-signifiante, alors que c'est la même notion qu'utilise Bion pour caractériser, dans une perspective tout à fait semblable, la matière du rêve ; celle dans laquelle l'activité fantasmatique vient puiser pour construire les scénarii du rêve. Ne faut-il pas alors considérer que le monde interne puisse être tenté de s'enfouir dans cette masse plastique que constituent les images-mouvements que nous délivrent à foison les nouveaux médias pour mieux s'accaparer les signes sensibles capables de relancer les mouvements de subjectivation?

La mise à profit par le processus de subjectivation de cette matière figurable intrinsèque aux Computer Mediated Relations (CMR) et en particulier aux jeux vidéo, nous a été rendue particulièrement explicite à différents moments du travail thérapeutique mené avec Tom. Ce jeune patient su non seulement nous amener à reconsidérer nos a priori initiaux sur ces nouveaux modes de communication que sont les tchats, blog, skype, msn ou divers réseaux net, mais parvint également à nous convaincre que l'écoute et l'attention que nous pouvions porter à la description des différents types d'investissements susceptibles de se déployer sur ces nouveaux supports pourraient être précieuses à plus d'un titre : que ce soit pour déterminer dans quelle mesure ces nouveaux territoires pourraient appartenir à une nouvelle cartographie de l'espace psychique ou que ce soit pour définir si l'instauration de ce nouveau type d'investissement n'offrirait pas au clinicien une nouvelle voie d'accès à la réalité interne qui aurait pour caractéristique d'être beaucoup plus diffuse et mouvante.

\section{L'UNIVERS ONIRICO-VIRTUEL DE TOM}

Longtemps nous avons cherché à rendre compte de la dévotion de Tom à l'égard de ces objets électifs qu'étaient pour lui sa console ou son ordinateur. En effet, il se devait de respecter scrupuleusement un rituel du coucher qui lui imposait de consacrer au moins quelques instants d'attention à sa console vidéo, le temps de s'assurer
7. G. Deleuze,

L'image-temps, Paris, Minuit, 1985, p. 44. 
qu'elle était en bonne place et de lui offrir précautionneusement un dernier coup de chiffon. Longtemps le côté impérieux de toute cette déférence conservera pour nous un aspect énigmatique, jusqu' au jour où il finira par nous dire que sa console représentait ni plus ni moins que «la moitié de son cour». Aussi cherchions nous à appréhender quel sorte de magnétisme exerçait sur lui les consoles et jeux vidéo, tout en nous efforçant de préciser le rôle que pouvaient jouer ces nouvelles prothèses psychiques dans le travail thérapeutique mené avec lui. Celles-ci apparaissaient en effet être convoquées pour pallier à des difficultés personnelles qui se traduisaient par des angoisses anxio-dépressives conjuguées à des états de tristesse, des insomnies, et jusqu'à l'envie de mourir.

Là encore, nous fûmes grandement aidé par Tom, qui d'une façon spontanée et naturelle prit l'habitude d'amener lors des séances, du matériel lié à ses propres découvertes dans la sphère des jeux vidéo. Ainsi, au fil des séances des interférences et des entrecroisements surprenants vont se produire entre les scénarii des jeux vidéo et la trame narrative plus personnelle de cet adolescent. Ce sont donc ces glissements, ramifications ou collusions entre séquences vidéo et rêves ou fragments de rêve dans les jeux qui nous aurons particulièrement troublés. Ce sentiment d'inquiétante étrangeté, sera encore accentué lorsque des phénomènes de superpositions étranges vont s'établir entre des figures héroïques présentes dans certains jeux vidéo et des figures identifiantes qui alimentent sa propre sphère fantasmatique.

Très vite, Tom s'était plu à me décrire les séquences de combats et les stratagèmes qu'il mettait en place pour déjouer les ruses de la pléiade d'assaillants qui se succédaient face à lui sur l'écran. Les idées préconçues qui étaient les miennes, me laissaient craindre un aspect répétitif de ses récits. Or, ces craintes furent systématiquement mises en brèche par la façon singulière et passionnée qu'il avait de me retracer le fil narratif des jeux qu'il découvrait. Plus le temps passait, plus je le laissais, voire même l'incitais à me préciser les scénarii et les enjeux sous-jacents, lui-même se montrant parfois surpris que je puisse octroyé quelque intérêt à ce domaine exécré et honni par ses parents.

Parmi les nombreux scénarii qu'il ait pu nous raconter l'un d'eux aura tout particulièrement retenu et éveillé notre attention, dans la mesure où il faisait directement écho aux angoisses d'être précipité ou de sombrer dans un trou noir ; angoisses que Tom relatait en évoquant notamment ses cauchemars. 


\section{L'HISTOIRE AMNÉSIQUE DE «TREIZE »}

Ce jeu se déroule autour d'un personnage central, Treize, qui doit composer avec une amnésie totale de son passé. La réalité le rattrape très vite puisque avant même de comprendre ce qui lui est arrivé, il s'aperçoit que des tueurs à gages sont à ses trousses. Confronté à cette situation énigmatique, le seul indice qu'il possède susceptible d'éclairer son passé est un tatouage avec le n XIII inscrit à même son corps. Treize ne tardera pas à redécouvrir ses aptitudes à rivaliser et à se défendre face à ses ennemis, qui le soupçonnent d'avoir assassiné un président de la république dans sa voiture. Mais cet élément lui échappe initialement puisqu'il est confronté à un black-out total de son passé.

En dehors des phases classiques de combat, ce jeu possède la particularité de mettre en scène des séquences où le joueur est transporté dans les cauchemars du héros lui-même. Tom m'expliquera alors que le passage dans l'univers onirique de XIII se traduit même par un changement au niveau figuratif avec le passage de la couleur au noir et blanc, les cauchemars de Treize étaient ainsi transposés en noir et blanc sur l'écran. L'ensemble de ces effets participera à amplifier les mouvements identificatoires de Tom au héros de l'histoire et l'amènera à vivre avec une intensité émotionnelle redoublée les angoisses et craintes de ce personnage. Etat de fait qui sera encore accentué lorsque le jeu l'amènera à accompagner Treize dans les méandres de ses cauchemars. Celui-ci se verra par exemple dans l'obligation d'explorer les recoins obscurs d'un bateau. Son parcours est évidemment jalonné de pièges et de mauvaises surprises. En effet, sur ce bateau, il convient de se déplacer avec les plus grandes précautions puisque les zones d'ombre sont synonymes de la présence de trous noirs dans lesquels Treize risque de tomber et de disparaître corps et âme. Le jeu suppose bien sûr d'éviter toutes ces zones piégeuses, sans quoi le héros et le joueur abandonnent une vie, les jeux vidéo ayant cet avantage incomparable d'octroyer plusieurs vies aux protagonistes.

Tout en écoutant Tom nous raconter ce scénario et me livrer moult détails au sujet des cauchemars de Treize, je voyais poindre sur son visage un sourire en coin, malicieux nous laissant clairement entendre qu'un insight s'était produit de son côté, rendant du même coup superflue toute interprétation de notre part. Cette séquence nous laissait néanmoins bien percevoir la façon dont Tom pouvait vivre les séances : que ce soit par rapport à l'intensité émotionnelle de la relation transférentielle que le passage de la couleur au noir et blanc venait traduire ou que ce soit par rapport aux résistances 
et précautions rendues nécessaires par la crainte que le dispositif thérapeutique, au même titre que les recoins obscurs du bateau, dissimule quelques mauvaises surprises ou pièges capables de lui faire vivre une affliction pesante, voire une angoisse insurmontable comme celle suscitée par les trous noirs. Mais sans doute, était-ce là aussi l'expression d'une phobie banale à mon égard.

\section{ASSOCIATIONS SUR LA SCÈNE ANALYTIQUE}

Pour permettre au lecteur de mieux appréhender les aspects latents du récit de Tom, nous reprendrons brièvement le fil des associations de la séance. Son propos s'était tout d'abord attardé sur les problèmes qu'il rencontrait au collège avec son prof d'histoire... Son « pire ennemi », disait-il !

Ce sujet s'épuisant assez vite, et peut être déçu par le manque de sollicitude de notre part, il prolongeait ses dires avec le récit de cauchemars qui l'avaient agité les nuits précédentes, Tom nous décrivant alors le déroulement des trois séquences oniriques suivantes :

- La première concernait électivement la sensation de tomber dans un trou noir ; il m'expliquait que cela lui était arrivé deux fois de le vivre le matin juste avant le réveil et que son père arrivait juste au moment où il allait s'écraser. Malgré ce sauvetage inespéré, il se sentait totalement épuisé, harassé, au point d'ailleurs d'en venir à douter qu'il puisse réussir à se lever tellement la tête lui tournait.

- Il se souvenait ensuite d'un autre cauchemar où il assistait impuissant au spectacle de sa console PS2 se brisant et tombant en morceaux alors qu'il jouait tranquillement à son jeu favori : « $13 »$.

- Enfin un troisième cauchemar particulièrement douloureux lui aussi où il se sentait condamné et rejeté par sa famille qui le trouvait indigne d'une reconnaissance quelconque tellement il se confortait dans un registre insignifiant, dans la fainéantise. Tête basse et honteux, il ne savait plus quel échappatoire emprunter, en dehors de la fuite.

De prime abord, assez peu de matériel s'énonçait directement autour de ses rêves. Cela s'expliquait, en partie, par le caractère peu construit de ces cauchemars où les registres originaires et primaires prenaient le pas sur les possibilités de secondarisation du travail du rêve ; les éprouvés et notamment les éprouvés d'angoisse annihilant la trame narrative. Ce constat devait être cependant relativisé dès lors que l'ensemble des associations de la séance pouvait être entendu comme l'expression des matériaux latents de ces rêves. Dans cette perspective, non seulement, il devenait très clair que les éléments 
puisés dans le scénario de Treize participaient étroitement du contenu latent des cauchemars de Tom, mais il apparaissait également que Tom avait largement puisé dans le creuset de l'histoire de Treize, au point d'avoir l'étrange sensation d'une intrication entre le jeux et les rêves et de ne plus savoir s'il fallait penser que le jeux rentrait dans le rêve ou si à l'inverse c'était le rêve qui pénétrait dans le jeu.

\section{REDOUBLEMENT ET RÉDUPLICATION ENTRE RÊVES ET JEUX}

Le redoublement et la réduplication entre les cauchemars de Treize et ceux de Tom amèneront ce dernier à nous faire part d'un sentiment de déjà-vu responsable chez lui d'un certain trouble qu'il aura du mal à dissiper. Nous même étions quelque peu saisi, en même temps que circonspect et dubitatif quant à la façon d'envisager ces collusions entre scène fantasmatique et scène virtuelle. Nous restions avec un sentiment très partagé puisqu'à certains égards l'immixtion de l'univers des jeux dans l'univers onirique pouvait être appréhendée comme un procédé auto-thérapeutique, où les jeux seraient utilisés pour re-dynamiser un auto-érotisme défaillant, réintroduire un espace transitionnel là où le vécu d'effroi serait omniprésent et envahissant ; voire pour lui permettre de tisser des fragments imaginaires capables de border ces trous noirs qui engloutissent tout pouvoir de représentation. A d'autres égards, ces interpénétrations constantes du jeu dans le rêve et du rêve dans le jeu pouvaient apparaître inquiétantes, laissant craindre d'être davantage hors symbolisation comme le signifiait Lacan en parlant du rêve dans le rêve comme proto-typique d'une irruption du Réel dans l'espace onirique. Cela nous amenait alors à nous demander si l'écran vidéo n'était pas sollicité pour assumer transitoirement la fonction d'écran et de filtre qui revient habituellement à l'hallucination négative, comme si le rêve faisait appel à l'écran vidéo pour contrecarrer une défaillance de son propre support projectif. Toujours pour tenter le domptage des composantes pulsionnelles, le travail du rêve semblait également solliciter la trame narrative, comme les mythèmes basiques de ces jeux, afin de trouver un étayage minimal.

Notre perplexité nous incitait par ailleurs à nous contenter d'interventions prudentes qui privilégiaient avant tout l'établissement de certains liens par exemple en lui signifiant que ses cauchemars devaient être envisagés ensemble pour bénéficier d'un autre éclairage, le troisième de ses cauchemars étant susceptible de donner du sens aux deux autres. En l'occurrence, il s'agissait de 
lui faire entendre que le moment où il se sentait rejeté, banni, abandonné par sa famille rendrait partiellement compte de ses éprouvés dans les deux autres cauchemars où il ressentait avec force le sentiment d'avoir le cœur en morceau, le cœur brisé et la peur de tomber dans le vide. Cette suggestion l'amènera d'ailleurs aussitôt à préciser que le troisième cauchemar mettait en présence toute sa famille et notamment ses parents, son grand-père et ses oncles paternels, dans un décorum qui s'apparentait à une sorte de tribunal où les sentences plus impitoyables les unes que les autres étaient énoncées à son encontre. Ainsi était-il jugé coupable de paresse et de fainéantise inacceptable, de bêtise mettant en relief un défaut d'intelligence particulièrement dommageable et irrémédiable. Bref, désigné coupable et impardonnable, il ne lui restait que la solution de prendre la fuite pour faire cesser cet assaut de critiques et ces reproches qui confinait à la honte et à l'humiliation.

Cette tonalité très surmoïque - un surmoi cruel et tyrannique - que l'on voit surgir à l'occasion de ce cauchemar sera de plus en plus présente au fil du travail thérapeutique dans les scénarii relatés par Tom, au point d'ailleurs qu'il en viendra à se plaindre de ses rêves qui "lui font régulièrement une mauvaise vie et gâchent ses journées »! L'évolution de l'activité onirique attestera cependant d'une certaine humanisation de l'instance surmoïque pour la rendre progressivement moins féroce ou cruelle. La sévérité et le sadisme de cette instance rendaient compte d'éléments transférentiels que l'on sent poindre sous les traits du professeur d' histoire. Nous endossions ainsi tout d'abord le rôle redoutable et redouté de « Maître sorcier » l'incitant à se faire « apprenti historien », en l'amenant à travailler sur son histoire, une histoire dont on peut penser qu'il se ferait volontiers amnésique. Au demeurant, il restait difficile de préciser si cette amnésie concernait prioritairement Tom, au même titre que Treize, ou si celle-ci engageait avant tout l'attitude du père de Tom peu disposé à se remémorer certains de ses souvenirs d'enfance. Quelques entretiens menés conjointement avec Tom et son père, nous laisseront très vite deviner quelle place envahissante occupaient dans l'histoire paternelle des souvenirs qui convoquaient à nouveau la présence d'enseignants « bourreaux », dont les attitudes violentes et sadiques avaient émaillé tout son parcours scolaire ; cette vie scolaire faisant largement écho à la vie que lui menait son propre père pour le moins autoritaire et coercitif.

De fait, c'est toute la violence - et la jouissance masochiste paternelles contenues à l'égard des enseignants sadiques qui l'avaient régulièrement humilié dans sa jeunesse, mais aussi l'agressivité et la rage à l'égard des collègues lui créant des difficultés dans son cadre professionnel, ou encore la haine à l'encontre de son père vénéré qui 
semblaient réactualisées, et comme agies dans les comportements de Tom. Une violence mobilisée pour enfin trouver les moyens de restaurer ce père humilié et le faire advenir, via la relation transférentielle et sa duplication dans des scènes puisées dans l'univers des jeux virtuels, en tant que «père aimant » indispensable à la consolidation du narcissisme primaire.

\section{UNE NOUVELLE VOIE ROYALE?}

Nos interrogations initiales cherchaient à préciser dans quelle mesure l'investissement déployé par Tom sur certains jeux vidéo serait à même, - au-delà des risques réels de capture imaginaire, de captation scopique - de participer à une relance du processus de subjectivation. L'attribution d'une valeur subjectivante à ce type d'activité n'allait pas de soi dans la mesure où le risque de collage identificatoire ou de capture imaginaire, capable d'aliéner toute créativité personnelle, n'était pas négligeable. La tentation était donc grande de nous cantonner dans un positionnement où nous aurions surtout mis en avant que de tels investissements traduisaient pour l'essentiel une résistance narcissique à l'investissement d'objet.

Or, au-delà de ces risques, ces nouveaux supports de jeux et de communication semblent avoir pu favoriser pour Tom une reconnaissance et une différenciation des imagos psychiques. En effet, "face aux bouleversements de son monde interne, aux surinvestissements des objets internes, aux régressions pulsionnelles qui les affectent, et à la confusion des imagos qui peut en résulter, l'adolescent a besoin des objets externes pour s'étayer et pour leur faire jouer le rôle d'organisateurs de son monde interne. C'est ce balancement des investissements du Moi de l'adolescent entre son monde interne et ses objets d'investissements externes qui lui permet une relative maîtrise de la situation et une sécurisation narcissique. ${ }^{8}$ En même temps que des possibilités de réassurances narcissiques conquises par l'entremise de ces nouveaux supports externes, Tom trouvait également via le prisme des jeux vidéo la possibilité d'une figuration de ses imagos contribuant même au maintien d'un relative différenciation de celles-ci ; les manifestations d'angoisse atteignant leur seuil maximum dans ces moments d'indifférenciation. Il n'est évidemment pas possible d'exclure l'hypothèse inverse dans des conditions autres et notamment en l'absence d'un travail psychothérapique de Tom ; c'est-à-dire des conditions où l'absence d'élaboration personnelle aurait pu favoriser un recours addictif au jeu vidéo et participer d'une condensation et
8. Ph. Jeammet,

Réalité externe et réalité interne. Importance et spécificité de leur articulation à l'adolescence, Rev. Franç. Psychanal., 3-4, 1980, p. 498. 
9. S. Missonnier,

Pour une

psycho(patho)logie du virtuel quotidien, in Le virtuel, la présence de l'absent, ouvr. coll. sous la direction de

S. Missonnier et H. Lisandre, Paris, EDK, 2003, p. 156. d'une cristallisation des imago, au point d'en accentuer de façon massive la dangerosité. Loin d'être créatif l'usage des «CMR » aurait pu alors renforcer une sorte d'évitement paranoïde d' angoisses de persécution, celles-ci trouvant "dans le cyberspace l'illusion d'externaliser et de contrôler leurs conflits internes. ${ }^{9}$ Le mirage de s'extraire d'une compulsion de répétition serait ainsi auto-entretenu par la fascination qu'exerce l'univers virtuel. En d'autres termes, les échanges médiatisés par l'ordinateur ne feraient que révéler et amplifier les potentialités défensives schizoparanoïdes de Tom, avec le risque d'induire ou d'accentuer un clivage aliénant et une relation d'objet partiel projective.

$\mathrm{Si}$ ce risque a pu exister et ne devait pas être négligé, la configuration qui était celle concernant Tom semble davantage correspondre à un aménagement momentané des défenses et de l'espace psychiques. Les processus adolescents engendrent par euxmêmes chez tout sujet des mouvements paranoïdes et schizoïdes - en lien avec ce phénomène de condensation des imagos - et nécessitent du même coup, dans l'attente d'une reventilation des imagos, le recours à la création d'un espace psychique élargi. Or, si l'homothétie entre adolescence et scène virtuelle apparaît si évidente, c'est en grande partie via les superpositions qui s'opèrent entre l'aménagement d'un «espace psychique élargi » (dans l'acception que lui donne $\mathrm{Ph}$. Jeammet), et le phénomène de périphérisation ou d'externalisation de la vie psychique induit par la pratique des CMR.

\section{LA VIDÉO COMME «ESPACE PSYCHIQUE ÉLARGI »}

Au même titre que d'autres objets externes, les jeux vidéo et les personnages centraux qui en animent la trame narrative ont pu jouer pour Tom un rôle supplétif d'une des fonctions de l'appareil psychique. Véritable prothèse psychique, ils purent ainsi tour à tour occuper la fonction de Moi auxiliaire ou d'instance idéale (par exemple de double idéal de lui-même), ces fonctions étant sollicitées pour maintenir ou réamorcer un travail de subjectivation menacé de stase et de paralysie. En ce sens, l'investissement porté sur les jeux vidéo aura pu correspondre au déploiement et à l'aménagement d'un " espace psychique élargi ». Le recours à l'espace, à l'utilisation de l'espace, et notamment à l'espace virtuel, viendrait ainsi suppléer l'impossibilité pour le sujet de s'appuyer sur une temporalisation psychique suffisamment fiable pour instaurer une certaine continuité d'être. L'adolescence est, de fait, souvent 
synonyme d'un temps disjoint qui prive le sujet d'un sentiment de permanence, le laissant du même coup en proie au vide ou à l'ennui. A ce titre, l'obligation de maintenir que ce soit dans les combats, les courses ou autres poursuites, un niveau d'activité et de vigilance soutenu participe non seulement d'une stimulation de l'activité psychique, mais également d'une séquentialisation de celle-ci. L'activité rythmique imposée par la pratique des jeux se révélerait ainsi apte à contrebalancer momentanément l'atonie et la morosité qui seraient la marque d'une tonalité dépressive, mais aussi de l'instauration d'une relation fétichique aux objets qui participent grandement du sentiment d'habiter un univers où tout se fige, privé de toute temporalisation vivifiante.

Parallèlement à cette recherche de séquentialisation de l'activité psychique, le sujet qui ne peut s'appuyer sur sa propre réalité interne est amené à contre-investir la réalité interne par le biais de la réalité externe, c'est-à-dire qu'il se trouve dans l'obligation de privilégier les phénomènes d'espacement sur les phénomènes de temporalisation. En d'autres termes, il s'agit d'investir le monde perceptivo-moteur au détriment de la réalité interne et du monde fantasmatique. L'investissement de supports ou d'espaces externalisés - comme on le voit à l'œuvre avec la création d'un espace psychique élargi participe donc de ce mouvement d'espacement (devenir espace du temps) avec pour corollaire l'effacement ou tout au moins la mise en retrait provisoire de la dimension fantasmatique au profit de l'activité de perception.

\section{CONCLUSION}

Nous entrons dans une aire où, comme le signifiait Deleuze, toute différence semble s'estomper entre la nature et l'artifice. Ainsi, ce que l'on nomme monde virtuel ne serait du point de vue de notre réalité psychique qu'une nouvelle modalité d'apparition de la réalité externe. En ce sens, le virtuel apparaît bien comme un moyen qui ne se limite pas à une capacité à reproduire la réalité mais aussi à l'étendre. Il suffit d'ailleurs de faire l'effort de se projeter dans quelques décennies pour se rendre à l'évidence d'une totale intégration à la réalité externe de ce monde décrété aujourd'hui virtuel ; il ira de soi pour les sujets des générations à venir que l'ensemble des produits "high-tech » qui font leur apparition au fil du temps intégreront leur environnement au même titre que la télévision, le téléphone, l'ordinateur intègrent notre réalité quotidienne sans qu'on puisse véritablement les démarquer de notre 
environnement « naturel »!

Ce mouvement d'extension de notre réalité externe, lié aux inventions techno-scientifiques, nous semble, simultanément aux avantages pratiques qu'il représente, répondre également à une nécessité interne. De fait, la démultiplication de ces prothèses psychiques ou organes artificiels pourraitbien participeràde nouvelles formes de subjectivation qui tentent de remédier à nos difficultés contemporaines qui touchent à la construction du narcissisme primaire et à la résolution de l'intégration psychosomatique. Il est classique d'évoquer l'idée d'extension narcissique concernant la relation à ces nouveaux simulacres technologiques. Il est peut être moins spécifié en quoi ceux-ci pourraient participer à un remaniement des modalités de symbolisation. En effet, si l'on accepte une vision très (trop) généraliste, les processus de secondarisation tels qu'on les a connus jusqu'à ces dernières décennies semblaient favoriser une prévalence du fonctionnement psychique tourné vers différentes formes de symbolisation plutôt idéatoires ou intellectualisantes. On ne peut, en effet, que prendre en compte l'évolution des représentants de la pulsion vers l'abstraction dans la mesure où la pensée et l'abstraction vont de pair et participent d'un pouvoir de maîtrise qui a pu faire la preuve de son efficacité dans le domaine de l'exploration du monde physique. Aujourd'hui, via le cyberspace et la profusion des signes qui le caractérise, il semble que ce soit préférentiellement le langage d'action ou les images motrices qui aient davantage d'impact que les processus de pensées. Il ne s'agit évidemment pas de postuler la fin ou l'extinction progressive de la logique signifiante, même si on peut craindre un appauvrissement du potentiel métaphorique véhiculé par le langage, mais de souligner la mise en retrait ponctuelle de la prédominance de celle-ci quand bien même elle reste incontournable pour penser la structuration psychique. Comme si une contestation collective implicite s'évertuait à dénoncer, sans l'exprimer sur un plan manifeste, une distanciation trop grande par rapport aux sources libidinales directes qu'organisait l'activité de penser sous le primat du langage. En effet, «la pensée doit obéir à la double tâche de s'éloigner suffisamment des dérivés pulsionnels où elle prend naissance sans cesser de maintenir le contact avec ses racines affectives qui lui donnent son poids de vérité. Il y a là une structure paradoxale de la pensée en psychanalyse qui ne peut être

10. A. Green, La double limite, in La folie privée, Paris, NRF, 1990, p. 298. dépassée. ${ }^{10}$

$\mathrm{Si}$ le socle de nos civilisations repose en grande partie sur les acquis et progrès rendus possibles par l'élévation du fonctionnement psychique jusqu'à ce mode de représentance que sont les signifiants et leur organisation en chaînes signifiantes, Winnicott a su montrer comment la prévalence de ce mode idéatoire pouvait avoir des 
contreparties moins favorables dès lors que l'esprit, l'intellect fonctionnent sur un mode totalement désolidarisé de la réalité interne. Il en découle d'une part l'achoppement de l'intégration psyché/soma dans la mesure où la pensée requiert ce mouvement contradictoire de séparation d'avec le corps et de constante réunification avec lui. Et il en résulte, d'autre part, une fixation du fantasme, ce dernier étant à appréhender dans la terminologie winnicottienne comme un élément strictement défensif qui doit être situé aux antipodes justement de la réalité interne ou du rêver. Dès lors, les espaces transitionnels du dedans (entre l'inconscient et le conscient-préconscient) et du dehors (l'espace potentiel) ne fonctionnent plus comme des aires de compromis que ce soit entre l'inconscient et la censure pour le premier ou entre le monde interne et le monde externe pour le second, mais à l'inverse participent à figer la vie psychique.

Or, c'est précisément au niveau d'une possible restauration de la fonctionnalité de cette double limite (ICS/CS ; dedans/dehors) que le recours aux CMR et en particulier aux jeux vidéo nous apparaît posséder certaines vertus. S'il est malvenu d'avancer des conclusions qui auraient une portée générale à partir des seuls éléments cliniques exposés, ceux-ci nous incitent néanmoins à nous défaire d'une perception d'emblée péjorative à l'égard des investissements que les patients peuvent déployer dans la sphère du monde virtuel. C'est notamment le cas des idées préconçues qui accolent immédiatement la notion d'addiction - même si celle-ci mérite d'être explorée - dès lors qu'il est fait référence aux échanges médiatisés par l'ordinateur ou d'autres supports comme les consoles vidéo.

En effet, concernant ce qui se joue plus directement sur la scène analytique, l'évocation des aspects liés au CMR pourrait - eu égard à ce que nous avons développé au sujet des nouvelles modalités de régulation de l'économie psychique - présenter l'avantage de placer le patient, et l'analyste avec lui, dans une certaine proximité avec les processus primaires. En se situant en position intermédiaire entre identité de perception et identité de pensée, ils parviendraient à opérer une réduction du clivage que Freud postulait entre un pan de l'activité psychique gouverné par le principe de réalité (l'activité de conscience) et un autre uniquement soumis au principe de plaisir (l'activité fantasmatique). Les CMR pourraient ainsi constituer un remède transitoire à l'écartèlement qu'à à subir le Moi lors de certaines phases existentielles comme la période adolescente. 


\section{Philippe Givre - Télétechnologies et nouvelles configurations psychiques}

Résumé : Productrices de suppléments psychiques artificiels, les télétechnologies participent non seulement de la construction subjectale des individus, mais contribuent également à l'émergence de nouvelles configurations de la vie psychique. Le remodelage de l'espace psychique et des processus secondaires qu'elles induisent peut être mis en relation avec la place prévalente qu'elles octroient au langage d'action et aux « images-mouvement» (cf. Deleuze). En effet, les proximités et les affinités qui se trament entre images-mouvements dans les jeux vidéo et les Darstellung qui constituent la matière fantasmatique ou le matériau des rêves, occasionnent des phénomènes de collusion - glissements de la scène virtuelle sur la scène onirique et vice-versa - qui s'avèrent, dès lors que l'on y prête quelque attention, propices à réduire l'écartèlement qu'à à subir le Moi lors des différentes phases de maturation subjectives.

Mots-clés : Darstellung — Langage d'action — Jeux vidéo — Imagesmouvement — Processsus secondaire — Télétechnologie — Virtuel.

\section{Philippe Givre - Teletechnologies and new psychic patterns}

Summary : Teletechnologies, which produce artificial psychic supplements, are not only part of the subjectal construction of the individual, but also contribute to the appearance of new patterns in psychic life. The remodelling of psychic space and the resulting secondary processes can be related to the prevalence given by teletechnologies to the language of action and «movement pictures» (cf. Deleuze). Indeed, the relations and affinities which are created between movement pictures in video games, and the Darstellung which constitute fantasy material or the basis of dreams, cause phenomena of collusion, shifts from the virtual scene to that of the dream, or vice versa - which, when attention is given to them, prove suitable for reducing the contradictory pressures which the Ego has to experience during the various subjective phases of maturation.

Key-words : Darstellung — Language of action — Video games - Movement pictures — Secondary process — Teletechnology — Virtual. 